\title{
NUMERICAL SIMULATION OF MICROWAVE SCATTERING FROM SEA ICE BASED ON THE FINITE ELEMENT METHOD
}

\author{
Xu Xu, Anthony P. Doulgeris, Frank Melandsø, Camilla Brekke \\ Department of Physics and Technology \\ UiT The Arctic University of Norway \\ Tromsø, Norway
}

\begin{abstract}
In this paper, a 2-dimensional scattering model for sea ice based on the Finite Element Method (FEM) is presented. The scattering problem is formulated following a physicsseparate strategy. The wave in the air domain is expressed by the scattered field formulation, while the wave in the sea ice domain is expressed by the total field formulation. The two separate physics and formulations are coupled through the boundary conditions at the air-sea ice interface. The proposed FEM is tested for simulating the radar cross section (RCS) of homogeneous sea ice at $\mathrm{C}$ and L bands. By comparing the results of the FEM with the Small Perturbation Method (SPM), good agreements are achieved.
\end{abstract}

Index Terms - Scattering model, sea ice, the Finite Element method, radar cross section

\section{INTRODUCTION}

As a critical component of the earth, sea ice influences wildlife, human activity, shipping navigation and natural resources exploration in Polar Regions and is highly sensitive to global climatic changes. Moreover, there is a dramatic decline of the Arctic sea ice over the past few decades [1], which increases the demand for reliable detection and characterization of sea ice.

The technology of high resolution Synthetic Aperture Radar (SAR) has been extensively used to monitor sea ice due to its all-day and all-weather capabilities. To better interpret sea ice from SAR, the link between physical parameters of sea ice and SAR signature needs to be built. Electromagnetic (EM) Modeling studies can be undertaken to simulate the expected backscattering from hypothetical sea ice. Through models, the measured SAR signature of sea ice can be related to its physical properties. And we can study which parameters are most sensitive to SAR signature.

In this study we describe a 2-dimendisonal numerical model for calculating scattering from sea ice based on the Finite Element Method (FEM). The FEM is a numerical technique for finding approximate solutions to Partial Differential Equations (PDE) by dividing the whole computional domain into subdomains of simple geometry called finite elements. Through the FEM, the PDE problems can be translated into a set of linear algebraic equations [2]. The FEM has been used for numerical modeling of physical systems in a wide variety of engineering disciplines. Recently, The FEM has been applied in calculation of radar scattering from soil [3]. But to the best of our knowledge, it has not been studied in sea ice scattering. The FEM has a flexible meshing procedure, which makes it well suited to heterogeneous structures and inhomogeneous volume scattering [4].

The paper is organized as follows: Section 2 details the procedure of the FEM for simulation of sea ice scattering. Experimental results are given in Section 3 and conclusion in Section 4.

\section{BASIC METHOD}

\subsection{Domain truncation with PML}

Before formulating the scattering problem with FEM, we need to use a boundary conditon to terminate the computational domain. An ideal boundary should absorb all outgoing waves at any incident angle with no reflection. Here a Perfectly matched layer (PML) is used to truncate the domain. Actually the PML is not a boundary condition, but rather a domain that we add along the exterior of the model. Inside the PML layer, the conductivity has a parabolic profile that increases from the inner boundary to the outside perfect electric conductor boundary [5]. A rectangle PML is set to surround air and sea ice domain, as is illustrated in Fig.1.

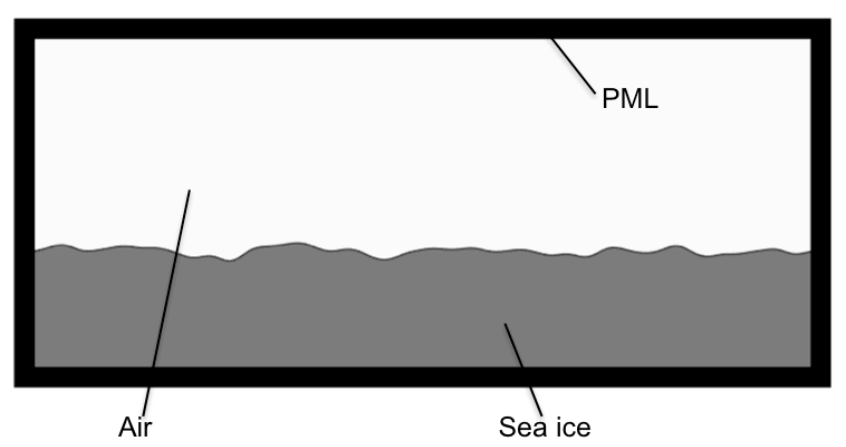

Fig 1. Physical model of scattering simulation for sea ice 


\subsection{Formulations of the FEM}

To implement finite element analysis of PDE, e.g. Electromagnetic wave equation in this study, there are two classic methods: the Ritz variational method and Galerkin's method. These two methods form the foundation of the FEM. Here we use Galerkin's method to solve the wave equation. An integral equation, known as a weak equation, is firstly built from the wave equation and test functions. If the whole computational domian is divided into a set of elements, we can get sets of integral equations. After incorporating available boundary conditions on the integral equations, all the equations are assembled and they form a sparse matrix which can be solved by using direct or iterative methods. In this study, the air and sea ice domains are discretized into triangles and the PML domain into rectangles. The system of equations are solved by the direct solver MUMPS based on LU decomposition [6].

The governing wave equation for the total wave or its corresponding weak form can be transformed into the scattered field formulation. This formulation supposes that the background field is known everywhere in the computational domain and the scattered field is the unknown to be solved. The difference between total field and scattered field formulations is how the incident wave is excited. For the total field formulaiton, the incident wave is excited by a source away from the surface of scatterers. As the incident wave propagates through the domain composed of elements, dispersion errors will be caused. For the scattered field formulation, the incident wave is known and introduced directly on scatterers.

In our problem, the scatterer is a layered media. If scattered field formulation is applied, we need to know the background field in the meidum which is hard to get. Consequently we propose a strategy using separate formulations. For the air domain the scattered field formulation is used and the total field formulation is applied on the sea ice domain. The two different physics mechanisms are coupled through the boundary conditions on the interface between air and sea ice. The details of the scattered field formulation and the total field formulation based on the FEM can be found in [7].

\subsection{Incident wave}

The incident wave needs to be defined for the formulations. The incident waves for calculation can be the tapered Gaussian wave or the plane wave. The implementation of a plane wave can cause errors due to reflections from the edges of a finite surface. An approach to solve this problem is to use periodic boundary conditons. Here we use the Thorsos tapered wave as incident wave [8]. This tapered wave can satsify the wave equation well by appropriate choice of the beam half waist.

\subsection{Simulation of sea ice}

A random rough surface is the result of a random process and can be characterized using the height distribution function (HDF) and the autocovariance function (ACF). The HDF describes height deviations from the root-mean-square (rms) height, while the ACF describes surface variation in the lateral directions accociated with the correlation length. We generate a Gaussian distributed rough surface for sea ice by using the method presented in [9].

The complex permittivity of sea ice can be represented by salinity and temperature data with the use of the Poldervan Santen-de Loor mixture model [10].

\subsection{Calculation of radar cross section}

Through the FEM, the scattered fields at near field region are calculated. The scattered fields at far field region are required to calculate the radar cross section. The near field to far field transformation is usually performed through the surface equivalence theorem. In this study, we use the Stratton-Chu formula to calculate the far field [11].

The Monte Carlo method is used to average the simulated field results because the surface roughness profile is random. A number of different rough surfaces with the same roughness parameters are generated. The scattered fields are calculated for every realization by the use of the FEM. Results are averaged to get the final mean radar cross section.

\section{EXPERIMENTAL RESULTS}

For validation of the FEM, the method is tested to simulate the scattering from homogeneous sea ice. In this case, the scattering only occurs at the sea ice surface. Results are compared with the commonly used method SPM. A typical dataset for newly formed sea ice is chosen and the permittivity is calculated as 5.5-0.2i. The rms height of sea ice surface and corrlection length are set as $0.002 \mathrm{~m}$ and $0.02 \mathrm{~m}$ which are within the realistic range.

The method is first tested for $\mathrm{C}$ band microwave (wavelength $\lambda=5.5 \mathrm{~cm}$ ). The surface is generated by connecting points that are $\lambda / 10$ apart and is $36 \lambda$ in length. By performing Monto Carlo simulations, a total of 200 sea ice surfaces are generated and the ensemble-averaged bistatic RCS is calculated for a fixed incident angle $40^{\circ}$. Fig 2 (a) and (b) show the results for $\mathrm{HH}$ and VV polarizations in comparision with SPM. Good agreements for both polarizations are achieved. It should be noted that the SPM results lack the realistic peak in the specular direction because it only accounts for incoherent scattering while the FEM can simulate both coherent and incoherent scattering.

The FEM is also appiled for $\mathrm{L}$ band microwave (wavelength $\lambda=24 \mathrm{~cm}$ ). Besides the frequency, all the other parameters are the same as $\mathrm{C}$ band test. The results are 


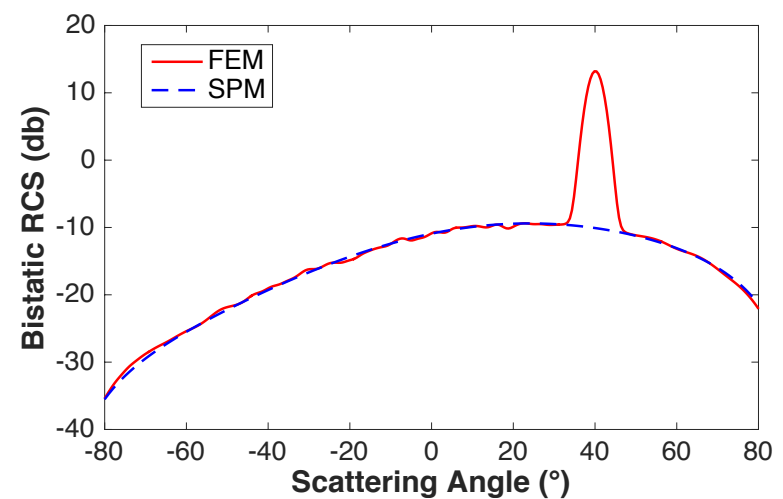

(a)

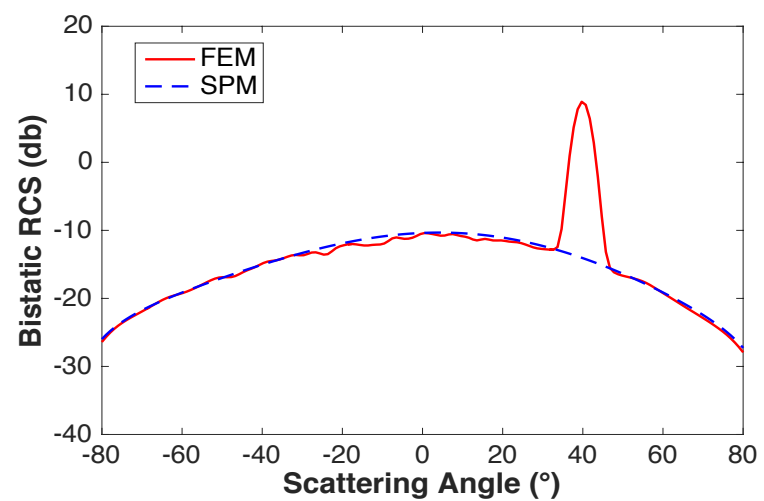

(b)

Fig 2. Bistatic RCS for (a) HH and (b) VV polarizaions simulated by FEM and SPM at C band

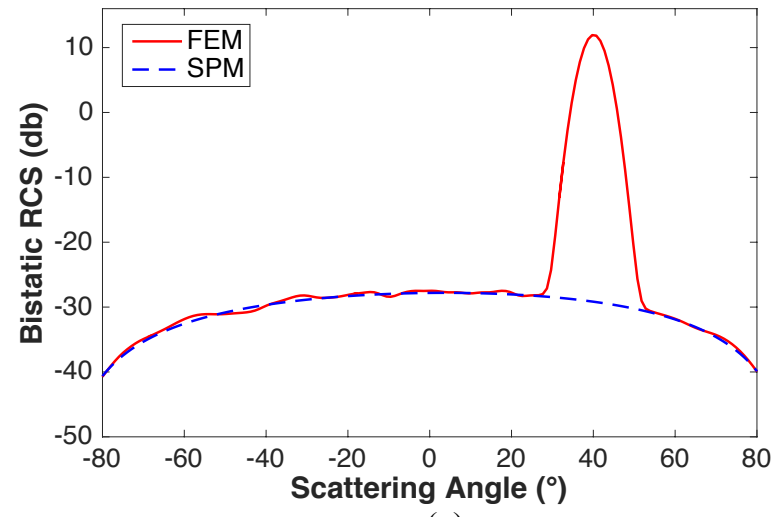

(a)

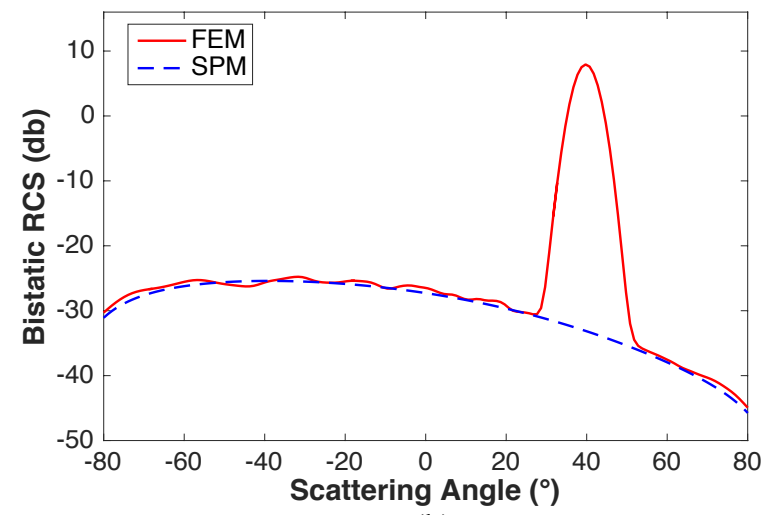

(b)

Fig 3. Bistatic RCS for (a) HH and (b) VV polarizaions simulated by FEM and SPM at L band

shown in Fig. 3 (a) and (b). Good agreements are also observed.

Compared with a 3-dimensional model, 2-dimensional modelling requires less computional time and memory. The above tests are performed on a MacBook Pro with 16 GB memory and the time for calculating the scattering of one surface instance is less than 30s. Parallel computing is also applied on Monte Carlo simulation, which decreases the total computational time effectively.

\section{CONCLUSION}

We developed a scattering model for sea ice based on the finite element method. To decrease the dispersion error, the whole computational domain is separated and expressed by the scattered field formulation and the total field formulation, respectively. The proposed scattering model shows good agreement with the SPM at different frenquencies.

The method can also be used to simulate the scatttering of medium with multiple layers, e.g. snow covered sea ice or inhomogeneous sea ice with a varying salinity profile. This will be studied in future work.

\section{REFERENCES}

[1] J. C. Comiso, "Large Decadal Decline of the Arctic Multiyear Ice Cover," Journal of Climate, vol. 25, pp. 1176-1193, 2012.

[2] G. Dhatt, E. Lefrançois and G. Touzot, Finite element method, John Wiley \& Sons, 2012.

[3] U. K. Khankhoje, J. J. van Zyl, and T. A. Cwik, "Computation of Radar Scattering From Heterogeneous Rough Soil Using the Finite-Element Method," Ieee Transactions on Geoscience and Remote Sensing, vol. 51, pp. 3461-3469, Jun 2013.

[4] K. F. Warnick and W. C. Chew, "Numerical simulation methods for rough surface scattering," Waves in random media, vol. 11, pp. R1-R30, 2001.

[5] E.M. Nassar, J.T. Johnson, and Robert Lee, "A numerical model for electromagnetic scattering from sea ice, "Ieee Transactions on Geoscience and Remote Sensing, vol. 38, pp. 1309-1319, May 2000

[6] MUMPS: a MUltifrontal Massively Parallel sparse direct Solver. Available from: http://mumps.enseeiht.fr.

[7] J.M. Jin, The finite element method in electromagnetics, John Wiley \& Sons, 2014. 
[8] E.I. Thorsos, "The validity of the Kirchhoff approximation for rough surface scattering using a Gaussian roughness spectrum," The Journal of the Acoustical Society of America, vol. 83, pp. 78-92, 1998.

[9] L. Tsang, J.A. Kong, K.H. D, and C.O. Ao, Scattering of electromagnetic waves: Numerical Simulations, John Wiley \& Sons, 2001.

[10] D. Polder and J. H. Van Santeen, "The effective permeability of mixtures of solids," Physica, vol.12, pp. 257-271, 1946.

[11] J.S. Lee, T.L. Song, J.K. Du, and J.G. Yook, "Near-field to far-field transformation based on stratton-chu fomula for EMC measurements," In Antennas and Propagation Society International Symposium (APSURSI), 2013 IEEE, pp. 606-607, IEEE, 2013. 